\title{
REVIEW OF TECHNOLOGY SOLUTIONS FOR KNOWLEDGE MANAGEMENT ${ }^{\mathrm{i}}$
}

\author{
Albena Antonova, Dr. Elissaveta Gourova, Assoc. Prof. Dr. Roumen Nikolov \\ Sofia University 'St. Kliment Ohridski’, Faculty of Mathematics and Informatics \\ IT Department, Bulgaria
}

\begin{abstract}
The present paper focuses on Knowledge Management (KM) as a new managerial discipline emerging in the last few years of the $20^{\text {th }}$ century. The main emphasis of the paper is on the technological solutions applied in the organizations at different stages of the KM life cycle. It makes a classification of the types of technologies described in the theory and practice based on the main KM processes. Finally, are presented survey data on the real application of various knowledge management technologies in the organizations.
\end{abstract}

Key words: knowledge management, knowledge management technologies, classification of knowledge management technologies

\section{INTRODUCTION}

The emergence of Knowledge Management (KM) concept is strongly related with the development of the information and communication technologies and the changes occurred in the organizations' structure, functions and management practices all over the world. Knowledge appeared to be essential asset of every company, and thus its gathering, preservation and better utilization turned to be of high importance for companies' development and success. Loosing of experts and their knowledge become very sensitive issue of companies. Thus, the strategy for collecting and managing the knowledge of employees is gaining high importance nowadays.

Historically, knowledge was a subject of discussions since the ancient Greek philosophers. However, the modern concept on knowledge management appeared at the end of the twentieth century. Due to the complex nature of knowledge and its intrinsic character, there is not accepted one single definition of knowledge management. The various definitions provided from researchers and practitioners literature explain KM mainly as the ability of the organization to adopt an unified approach to cope with its knowledge resources, in order to realize gain, interact better with the environment and increase performance. The distinction between the concepts of data, information and knowledge is discussed by several authors (Blumentitt, 1999; Bergeron, 2003). Davenport et al (1998) propose the understanding of data as 'set of discrete, objective facts about events', further 'information.... it has a shape: it is organized to some purpose' and finally knowledge that is the application of information to address specific situations in an organizational context. KM is related to several processes in the organizations, e.g. generation, storing, codification, representation, etc. of knowledge, which could highlight the diversity of technological tools described in the literature, and utilized for knowledge management in the organizations.

This paper provides an attempt for classification of the technologies which are used to facilitate the KM processes. Different ways of classification of KM technologies have been taken into account - from generalized classification considering only three KM processes (Ruggles, 1997), e.g. knowledge generation, codification and transfer, to much broader diversification of KM processes (Bergeron, 2003; Housel and Bell, 2001; O’Leary,1998). The authors are aware of the fact that present KM theory and practice are based on technologies and approaches developed in virtually every field of the computer science. At the same time, practical surveys show that $\mathrm{KM}$ is unique for every company, and the majority of the companies develop their KM strategies using a kind of combination of standard and company-made software tools (KPMG, 2004).

Having all this in mind, the authors have classified the available technological solutions according to the following KM processes in which they are applied:

- Generation of knowledge

- Storing, codification and representation of knowledge

- Knowledge transformation and knowledge use

- Transfer, sharing, retrieval, access and searching of knowledge

\section{TECHNOLOGIES CLASSIFICATION}

\subsection{Generation of knowledge}

Most authors start the analysis of KM processes with the generation of knowledge, which could comprise the activities for knowledge creation, acquisition and 
capturing. Similarly, the first group of technologies in this overview is related to the KM technologies used for generation of knowledge.

The Knowledge content generation tools include various authoring tools (Bergeron, 2003) - word processing editors, multimedia editors, graphics programs, image and sound editors, video editing systems, as the focus is put on time-saving and efforts-saving technologies facilitating the process of creation of relevant high-quality content.

Another technology allowing the generation of knowledge from data is knowledge discovery, defined in (O’Leary, 1998) as "nontrivial extraction of implicit, previously unknown, and potentially useful information from data." Knowledge discovery is a method that includes different tools and approaches to analyze both text and numeric data. As an example of a class of technologies for knowledge discovery could be mentioned data mining tools or the process of extracting meaningful relationships from usually very large quantities of seemingly unrelated data. Specialized data mining tools allow managers to perform competitive analysis, market segmentation, trend analysis, sensitivity analysis, and predictions based on information in the corporate database (Bergeron, 2003).

As technologies enabling the capturing of knowledge can be presented data capturing tools (Bergeron, 2003), getting the information accurately and efficiently into a machine-readable form. In general, the technologies used for data capture are defined by the source and in this field there are technologies as web data capture (public search engines), optical character recognition (OCR) technologies (for printed material), to convert printed text to machine-readable text. Besides working with text, speech, and images, data capture technologies can be applied to physical objects as bar codes identification technologies, and real-time location sensors supporting object tracking.

\subsection{Storing, codification and representation of knowledge}

Technologies and tools contributing to the effective storage, archiving and codification of the knowledge focus the attention on another important aspect in the Knowledge management process - the quality, quantity and accessibility and representation of the acquired knowledge.

At first place should be considered several technologies for storage of data, information and knowledge as databases, knowledge bases, data warehouses and knowledge warehouses, data mart and data repositories.

- The data warehouse is the main component of KM infrastructure. Organizations store data in a number of databases. The data warehousing process extracts data captured by multiple business applications and organizes it in a way that is meaningful to the business for any future references in the form of knowledge (Gupta et al., 2004). For example, data warehouses could act as a central storage area for organization's transaction data. Data warehouses differ from traditional transaction databases in that they are designed to support decision making rather than simply efficiently capturing transaction data. Typically, data warehouses contain multiple years of transaction databases stored in the same database. Data warehouses are not updated on a transaction-by-transaction basis. Instead, the entire database is updated periodically (O’Leary, 1998).

- Knowledge warehouses are another type of warehouses aimed more at qualitative data, than the kind of quantitative data typical for data warehouses. Knowledge warehouses store the generated knowledge from a wide range of databases including databases, data warehouses, work processes, news articles, external databases, web pages, and people. Thus, knowledge warehouses are likely to be virtual warehouses where the knowledge is dispersed across a number of servers (O’Leary, 1998).

- Data bases and Knowledge bases can be distinguished by the type and characteristics of the data stored. While in the database the data have to be represented in explicit form, in the knowledge-based systems it is possible the generation of knowledge that does not explicitly exist in the data base. By this way the data in knowledge bases can be incomplete, fuzzy, and with factor of uncertainty. As given by O'Leary (1998), the Best-practices knowledge bases typically, are generated using benchmarking activities designed to solicit the more effective and efficient way of doing things. After an organization has knowledge of best practices, they can be incorporated in the operation. Subsequently, the Lessons learned knowledge base contains three types of lessons: informational, successful, and problem. An informational lesson might describe standard procedures for example in case of emergencies. Successful lessons capture positive responses to crisis. Problem lessons provide examples of things that went wrong and potential ways to solve the problems.

- A specific database system, focused on much smaller scale is the data mart, representing a structured, searchable database system, organized according to the user's likely needs. Compared to a data warehouse, a data mart has a narrower focus on data that is specific to a particular workgroup or task. Both data warehouses and data marts typically are built with some form of database management system, which is a program that allows a knowledge worker to store, process, and manage data in a systematic way. A data repository, in contrast, is a database used as an information storage facility, with minimal analysis or querying functionality (Bergeron 2003). 
In order to provide a broader view on the technologies, allowing the knowledge preservation in the knowledge bases and knowledge warehouses, some knowledge codification and representation aspects are briefly discussed below.

- Human-readable knowledge is represented using a wide range of approaches in KM systems. In many situations, case-specific information appears to provide the appropriate level of representation required for users to make best use of the knowledge. Case-based reasoning system allows companies to take advantage of previous problems or cases and related attempts to solve them (Tiwana, 1999). In other situations where the information is largely declarative knowledge (like facts and assertions), text or rules might be used to represent the information and knowledge. For example, manuals, newsletters, and other similar types of knowledge are typically provided in a document, list, or rule format (O'Leary, 1998).

- One popular approach is to codify the knowledge in terms of rules. However, a wide range of researches in the field of knowledge codification (concerning the theories in Artificial Intelligence) during 80s and 90s concluded that knowledge can be partly representable or not representable using the rule-based approach. A number of models and formalisms are developed in the artificial intelligence field, in order to enable the better knowledge representation, as the most popular between them are: production models (rule-based models or if-then models), procedural model, semantic nets, frames, formal logical models (Gavrilova et al., 2000).

The knowledge organization technologies allow better arrangement and facilitate the knowledge retrieval. Typical knowledge organization technologies are $\underline{\text { taxonomies, repository indexes and directories }}$ (Housel and Bell, 2001). In enterprise KM systems, ontology specifications can refer to taxonomies of the tasks that define the knowledge for the system. According the definition of Gruber (2000), the ontology is an explicit specification of a conceptualization. Ontologies define the shared vocabulary used in the KM system to facilitate communication, search, storage, and representation (O’Leary, 1998).

- Topic maps technology (Coakes, 2003) is an advanced solution to the problem of structuring, storing and representing knowledge within a corporation. It is established an ISO standard, as an answer to the problem of coherent representation of relations between topics (or ideas) and associating those topics with actual documents (topic occurrences). However, topic maps are limited instruments when it has to be represented the knowledge of each employee within a corporation.

- $\quad$ Skill maps are an extension of topic maps, creating new structures for storing information about employees, their knowledge and their skills, and it is created by copying specified topic map objects and adding individual modifications, providing mechanisms to enhance searching knowledge repositories that can take into consideration the state of each employee's knowledge and skills. Both topic maps and skill maps technologies use ontology framework for structuring and representation of knowledge (Coakes, 2003).

- $\quad$ Controlled vocabularies enable creating information, archiving it for future uses, and communicating it to others and to computer systems. Not only must there be a common language and vocabulary, but there has to be a common taxonomy a description of the relationship between words. From a business perspective, controlled vocabularies are critical because they define the ease with which knowledge workers and managers can store and retrieve information in Knowledge Management tools. This controlled vocabulary is often implemented as a data dictionary - a translation program that maps or translates identical concepts that are expressed in different words or phrases into a single vocabulary (Bergeron, 2003).

- $\quad$ Content management software represents the convergence of full-text retrieval, document management, and publishing applications. It supports the unstructured data management requirements of KM initiatives through a process that involves capture, storage, access, selection, and document publication. Content management tools enable users to organize information at an object level rather than in binary large objects or full documents. The information is broken down by topical area and usually tagged via extensible markup language (XML). Both capabilities dramatically increase the opportunity for knowledge documents re-use (Gupta et al., 2004).

\subsection{Knowledge transformation and knowledge use}

After the knowledge has been acquired or "sourced," it normally cannot be used in its raw form and must be transformed in order to become a valuable knowledge asset and to facilitate its further application and re-use (Liebowitz, 1999).

Knowledge can be transformed in many ways, specifically to conform to the format of the target repository. Traditionally, knowledge has been collected, compiled, verified, validated, and organized by an "end user", who embeds it into intermediate products, new customer products, or educational programs.

Specific transformation is required when knowledge is designated for a knowledge-based system (KBS). In that case, it must be reconstructed (using epistemological rational reconstruction principles) in a representation that supports the particular reasoning method employed by the KBS. Such reconstructions include "crisp" or fuzzy rules to support rule-based 
reasoning, editing into case formats to support casebased reasoning (CBR) (Liebowitz, 1999).

Another solutions and technologies taking part in the $\mathrm{KM}$ are Business Intelligence technologies (BI). BI is defined as the conscious, methodical transformation of data from any and all data sources into new forms to provide information that is business driven and results oriented. It often encompasses a mixture of tools, databases, and vendors in order to deliver an infrastructure that not only will deliver the initial solution, but will incorporate the ability to change with the business and current marketplace. The purpose of $\mathrm{BI}$ is to transform from an environment that is reactive to data to one that is proactive. A major goal of the technology solutions is to automate and integrate as many steps and functions as possible, to provide data for analytics that are as toolindependent as possible (Biere, 2003). Three types of tools are referred to as Business Intelligence Tools: Multidimensional Analyses software, that gives the user the opportunity to look at the data from a variety of different dimensions; Query tools allowing the user to ask questions about patterns or details in the data; and Data Mining tools which automatically search for significant patterns or correlations in the data (Mertins et al., 2003).

Some of the most popular tools and technologies enabling effective knowledge use and retrieval include:

- The expert systems (ES) are among the most popular instruments for Knowledge management. The expert systems use their knowledge bases and user responses to guide the user to recommended solutions. The expert system asks the user questions, comes to recommendations, and can explain the logic for the decision. As with a human expert, the user can either just take the system's answer or can interrogate the expert to learn how or why the decision was reached. The expert system approach prompts the user with questions, rather than relying on them to ask the correct questions. Based on the data that the user provides, the best solution is found and presented. If there is more than one solution, they can be ordered by likelihood (Liebowitz, 1999).

Expert systems can be classified according to the specific area of application on the following subclasses: data and signal interpretation ES, diagnosis ES, design ES, prediction ES, planning and scheduling ES, interpretation ES, decision-support ES, complex-systems control ES, and instruction/training ES (Gavrilova et al., 2000).

- Other popular systems enabling the KM usage in organizations are the decision support systems that allow managers and other knowledge workers to make decisions by reviewing and manipulating the data stored. Many of the technologies discussed here can be applied to some form of decision support. Decision support tools are one way to disseminate best practices, using technologies such as expert systems, simulations, and statistical analysis tools to view or manipulate information stored in the corporate data warehouse. These tools include text summarizing utilities (programs that distill a paragraph from extensive documents), outline generators, statistical programs to analyze data, and decision tables to verify that every possible scenario has been considered (Bergeron, 2003).

- $\quad$ Enterprise resource planning (ERP), Enterprise resource managing (ERM) and Customer relationship management (CRM) applications could embed significant knowledge about the organization, customers, and suppliers (Bell, 2001). It has to be considered to what extend the knowledge available is explicitly represented for enquiry, modification and refinement, focusing mainly on the knowledge about the nature of the processes, organization structure and strategic plan. One of the key challenges to ERP packages is to be able to integrate the many different types of knowledge and present them to users in a meaningful way.

- Visualization tools can also facilitate the knowledge processing and re-use. Graphics and animations, when appropriately rendered, can decrease the time required for knowledge workers to grasp complex processes and allow non-experts to verify the accuracy of relationships that would have been unwieldy to describe with tables of data. For example, slide presentation with histograms and other images is usually much more appreciated and effective than a text presentation on the same subject (Bergeron 2003).

- Another KM class of tools is knowledge simulation - programs that mimic reality by animating complex processes. Simulations are especially useful to knowledge worker to provide them understanding of complex relationships, tables of numbers or equations. Simulations are an excellent means of exploring what-if scenarios in an interactive format because they can display complex processes in an easy-to-understand way. Simulation-based animations are especially good at imparting the dynamic relationship between variables. Examples of visualization tools range from three-dimensional graphic packages to simple pie chart and histogram output from spreadsheets and other traditional office programs (Bergeron, 2003).

\subsection{Transfer, sharing, retrieval, access and searching of knowledge}

The appearance of web technologies provides the Knowledge Management with very rich tools for knowledge access and transfer in a timely and machine-independent way. In fact, the Web offers a very powerful platform for tools supporting all stages of Knowledge management, allowing unprecedented degree of integration of different representational and communicational media. So, due to the quick 
development of the Internet technologies, stimulating collaboration, communication and dissemination services and their great impact on the Knowledge management, demonstrate the further potential for expansion of the web-based and web-enabled KM tools and services.

Related to Internet, Enterprise information portals (EIPs) are evolving as a single source of knowledgebased systems as they integrate access to knowledge and applications. EIPs provide a single point of entry to all the disparate sources of knowledge and information both within and outside an organization, usually through the Internet or a company intranet, allowing the companies to serve their customers, interact with business partners and suppliers, and offer employees access to online tools and the right content and knowledge for decision making. EIP functionality ranges from access to structured data used in classifying and searching unstructured data to support collaborative processes (Gupta et al., 2004).

According to Liebowitz (1999), groupware is an umbrella term describing the electronic technologies that support person-to-person and team collaboration. Examples of groupware include shared authoring tools, electronic whiteboards, desktop video conferencing (DVC), online forums, e-mail, online screen sharing, multimodal conferencing, electronic meeting systems (EMS), as well as systems for workflow and business process reengineering (BPR). Each of these technologies holds the potential to increase collaboration at a distance, reducing the cost of travel and the time knowledge workers waste in transit, as well providing flexible communication structures (connecting the people in new ways), increased communication speed, increased work performance and productivity, organizational memories recording and distributed knowledge context (Ruskov, 1998). Distributed workforces, information overload, and getting products to market as quickly as possible are just a few of the motivations pushing collaboration technology development.

Liebowitz (1999) discusses also the aspects of knowledge sharing technologies, as sharing knowledge is different from sharing information. Knowledge is not as easily duplicated as information; it is "sticky" and has a highly context-sensitive nature. The knowledge sharing can be synchronous or asynchronous (Tiwana, 1999). The formalized process of Web-based knowledge sharing is still very immature as it is important to realize that these tools (for example: communities of practice tools, digital staff directory, network workspace, awareness alerting service, yellow pages, mind-mapping tools) are just enablers and the focus on the knowledge sharing must be on softer social issues, motivation of the experts to share knowledge and the company culture.
As an example of popular knowledge sharing tool, the yellow pages are designed to store and distribute knowledge about the employees' skills (Mertins et al, 2003).

The interface tools enable the information retrieval, as to get it out of a database is as important as acquiring it. The interface as a point of human-computer communication defines the quality and efficiency of the interchange. The better the interface, the easier it is for knowledge workers and managers to interact with computer-based tools. Recently, tools and programs are developed, aiming to measure and improve the "usability" of the software interface in order to increase its productivity and human-computer interaction in general.

A wide range of well-known Internet search engines have been used to guide users to search and find information on the Internet. The search engines can be adapted to intranet environments for KM. The development of the search engines continues as they begin to become "knowledge navigators" performing more complex and detailed searches in wider data and knowledge bases and warehouses (Housel and Bell, 2001).

Intelligent agents, also known as bots or software robots, can be used to connect people to knowledge available on the Internet or intranets, relying on pattern matching technology to do their work. Intelligent agents are especially significant in acquiring information from the web, commercial databases, and intranets or corporate intranets. Intelligent agents, which can be resident on a PC or web based, accept user questions, convert the questions into the appropriate language, and then submit the questions to the appropriate search engines. The intelligent agents then remove duplicates, place the results in a standard format, and order the results. Most intelligent agents accept natural language input. The pattern matching technology that makes this possible is natural language processing (NLP). In addition to being useful in automatically formulating queries for search engines, NLP front ends can make database front ends more user-friendly (Bergeron, 2003).

\section{USAGE OF TECHNOLOGIES FOR KM}

The survey of KPMG (2004) provides a feedback on the utilization of different technologies for the aims of $\mathrm{KM}$ in Poland. The broadly used technologies are Internet (98\%), Intranet (79\%), Portals (75\%), document management systems (63\%), data warehouses (64\%), dedicated KM tools (57\%), decision support systems (51\%) and CRM systems (45\%). Respondents as well found Internet and intranet as the most effective technologies with regard to knowledge management (44\% and 46\% respectively). This could suggest that companies 
better assess widely used and recognizable technologies to which they have become accustomed than less known such as Expertise/Experts location systems (13\%) and artificial intelligence (5\%). A quarter of the respondents declared that $\mathrm{KM}$ technology has developed over time, $12 \%$ said they have a specially designed KM system, while 61\% stated that the KM system in place is a combination of both.

It is worth to mention also an earlier survey of KPMG (2000) in the UK and USA, where KM technologies were also much earlier adopted. Actually, the results are quite similar. 93\% of the respondents used the Internet to access external knowledge, 78\% used intranet, $63 \%$ - data warehousing or mining technologies, $61 \%$ - document management systems, $49 \%$ - decision support, $43 \%$ groupware and 38\% extranets.

\section{CONCLUSION}

This paper has provided a brief overview of KM technologies that can be deployed in all KM processes. It has been made clear that technology plays a vital role by KM, however, it should be emphasized that a successful KM implementation should be focused not only on technology, but on first place, on human and organizational issues. It should be well understood that KM tools are not a 'panacea', they are tools which help and support the knowledge processes in the organizations and all related activities of the managers and employees. KM requires a company wide strategy which comprises policy, implementation, monitoring and evaluation. Such a policy should ensure that knowledge is available when and where needed and can be acquired from external as well internal sources. Establishing a successful KM strategy requires overcoming a number of challenges. The successful implementation of $\mathrm{KM}$ in the organization depends also on the organizational processes and structures, business perspectives and organizational behavior issues. At the same time, the KM system should respond to the specific needs of the organization. Copyright (C)2006 Sofia University

\section{REFERENCES}

1.Bergeron B. 2003, Essentials of Knowledge Management, John Wiley \& Sons, Inc., Hoboken, New Jersey

2. Biere M., 2003, Business Intelligence for the

Enterprise, Prentice Hall, New Jersey

3. Blumentitt, R., Johnston, R., 1999 Towards a Strategy For Knowledge Management Technology Analysis \& Strategic Management (11)3 pp.287-300 4. Bukowitz, W., R.Williams, 1999 The knowledge management fieldbook, Prentice Hall, London 5. Coakes, E. (ed), 2003, Knowledge Management: Current Issues and Challenges, Idea Group Publishing, London
6. Gavrilova, T., Horoshevskii V, 2000, Knowledge Bases, Intellectual systems, Peter, Sankt Peterbourg (in Russian)

7. Gruber T, 2000: What is an Ontology?, URL: http://www-ksl.stanford.edu/kst/what-is-anontology.html

8. Gupta J. and Sharma S. (eds), 2004, Creating Knowledge Based Organizations, Idea Group Publishing, London

9. Davenport, T. H., L. Prusak, 1998, Working Knowledge: How Organizations Manage What They Know Harvard Business School Press, 2. Boston

10. Despres, C. Chauvel, D. (ed) 2000, Knowledge Horizons, The present and the promise of knowledge management, Butterworth Heinemann, USA

11. Honeycutt, J., 2000, Knowledge Management Strategies, Microsoft Press, Washington

12. Housel, T., Bell. A, 2001, Measuring and managing knowledge McGraw-Hill International edition, Singapore

13. KPMG, 2004, Knowledge Management in Poland 2004 - Research Report;

14. KPMG Consulting 2000, Knowledge management research report;

15. Lehaney, B., Clark S., Coakes E., Jack G., 2004, Beyond knowledge management, Idea Group Publishing, London

16. Liebowitz, J. 1999, Knowledge management Handbook, CRC Press LLC

17. Mertins, K., Heisig P., Vorbeck, J., 2003 Knowledge Management - Concepts and Best Practices, Springer Verlag, Berlin-Heidelberg, 18. O’Leary, D.E. 1998, Enterprise knowledge management

19. Tiwana, A. 1999, The Knowledge Management Toolkit, Prentice Hall

20. Ruskov, P. 1998, Groupware: Computer support of the groupware, University edition "Stopanstvo", Sofia (in Bulgarian)

21. Ruggles III, R. (ed) 1997, Knowledge management tools, Butterworth Heinemann, US

\footnotetext{
i This study is a part of a Knowledge Management overview, prepared in the framework of the pilot project TRAINMOR-KNOWMORE, funded by the European Program "Leonardo da Vinci”.
} 